\title{
Sufrimiento de mujeres en una ocupación urbana de São Paulo, Brasil: un desafío para el trabajo en atención primaria de la salud
}

\author{
The suffering of women living in an informal settlement \\ in São Paulo, Brazil: a challenge to the work of Primary \\ Health Care
}

${ }^{1}$ Autora de correspondencia. Licenciada en Psicología. Estudiante de maestría en Salud Colectiva, Faculdade de Ciências Médicas, Universidade Estadual de Campinas, São Paulo, Brasil. $\triangle$ (iD)

${ }^{2}$ Doctor en Salud Colectiva Profesor, Departamento de Salud Colectiva, Faculdade de Ciências Médicas, Universidade Estadual de Campinas, São Paulo, Brasil. $\Delta$ (iD)

Aline Messias Mota ${ }^{1}$, Rubens Bedrikow ${ }^{2}$

RESUMEN Entre los efectos sociales de la pandemia de COVID-19, el aumento de la pobreza, el desempleo y la desigualdad social en el país agravaron los problemas de salud, principalmente, de la población más pobre. Esta investigación buscó discutir potencialidades y limitaciones del proceso de trabajo en atención primaria de la salud, basado en la Estrategia de Salud Familiar. Para ello, entre enero y febrero de 2020, se realizaron entrevistas semiestructuradas a cuatro mujeres residentes de una ocupación urbana del interior del estado de São Paulo, Brasil. Se encontró que presentan sufrimiento relacionado con la precariedad de las viviendas, la provisoriedad, el aislamiento social y el silenciamiento. El modo en que se organiza la atención primaria de la salud y el proceso de trabajo de las y los profesionales dificulta tanto el acceso de una parte de la población a los servicios de salud, como la percepción de las y los profesionales sobre el sufrimiento de la población. Los resultados de la investigación indican la necesidad de una nueva evaluación y perfeccionamiento de la Estrategia de Salud Familiar.

PALABRAS CLAVES Población Urbana; Áreas de Pobreza; Mujeres; Atención Primaria de Salud; Salud Familiar; Brasil.

\begin{abstract}
Among the social effects of the COVID-19 pandemic, increased poverty, unemployment, and social inequality in Brazil have led to worsening health problems, especially in the poorest citizens. The purpose of this research was to discuss the potentialities and limitations of the work process in primary health care based on the Family Health Strategy. In order to do so, we conducted semi-structured interviews with four women living in an informal settlement in the interior of the state of São Paulo, Brazil, between January and February 2020. We found that the women experienced suffering in relation to issues such as housing precariousness, transience, social isolation, and silencing. The way in which primary health care is organized and professionals' work processes make it difficult for this population to access health services and for professionals to perceive their suffering. The findings of this research point to the need to reevaluate and improve the Family Health Strategy.
\end{abstract}

KEY WORDS Urban Population; Poverty Areas; Women; Primary Health Care; Family Health; Brazil. 


\section{INTRODUCCIÓN}

El objetivo principal de esta investigación es discutir potencias y limitaciones del proceso de trabajo en atención primaria de la salud, basado en la Estrategia de Salud Familiar, para percibir daños a la salud determinados por el aumento de la pobreza, la desigualdad social y la violencia de género en el país, agravados por los efectos sociales de la pandemia de COVID-19, en especial, el desempleo(1,2). $\mathrm{Si}$, por un lado, los dispositivos propios de la Estrategia de Salud Familiar, como el trabajo en equipos multiprofesionales, la clínica ampliada y compartida, el método clínico centrado en la persona, la construcción de proyectos terapéuticos singulares, el apoyo matricial y las visitas domiciliarias apuntan a avanzar hacia el deseado cambio paradigmático de la práctica clínica en atención primaria, por otro lado, se han revelado insuficientes para garantizar de forma consistente el acceso y la integralidad del cuidado a las poblaciones en situación de elevada vulnerabilidad $^{(3)}$. Con el propósito de fortalecer tal discusión, los autores estudiaron la situación de mujeres que viven en una ocupación urbana reciente en la periferia de un municipio del interior del estado de São Paulo, Brasil. Este artículo es el resultado de una investigación de maestría en salud colectiva titulada "Sofrimento de mulheres moradoras de ocupação urbana recente".

\section{Atención primaria de la salud y Estrategia de Salud Familiar en Brasil}

La atención primaria de la salud es el primer nivel de contacto y la principal puerta de entrada de varios sistemas de salud del mundo. Utilizan unidades de salud ubicadas lo más cerca posible del lugar donde viven y trabajan las personas, y buscan proporcionar un seguimiento longitudinal, continuo, de los individuos y las familias; resolver gran parte de las demandas y coordinar el cuidado en los diferentes niveles de atención, garantizando la integralidad de ese cuidado. Se espera que la proximidad espacial y temporal entre el equipo de salud y las personas vinculadas a ese equipo faciliten encuentros clínicos más democráticos, en los cuales la voz de las y los pacientes sea más valorada. En Brasil, las recomendaciones de Alma-Ata entraron en sintonía con principios defendidos por el movimiento de la Reforma Sanitaria sobre todo la universalidad y gratuidad del acceso y la participación popular. Los movimientos populares de salud, principalmente de las áreas periféricas de grandes centros urbanos del país, tuvieron una influencia decisiva para que la salud pasara a ser un derecho de la ciudadanía y un deber del Estado y para que la participación de la comunidad se volviera una de las directrices organizativas del Sistema Único de Salud (SUS), según consta en el Artículo 198 de la Constitución. Esa nueva política pública que, en teoría, valora las opiniones y deseos de la ciudadanía en los encuentros clínicos y de gestión, fue una conquista política de la lucha por la redemocratización del país.

La Estrategia de Salud Familiar, creada como programa en 1994, es considerada un elemento crucial para reorientar el modelo asistencial y una de las principales estrategias estructurantes de la atención primaria de la salud en Brasil. De acuerdo con el Ministerio de Salud, la Estrategia de Salud Familiar opera con equipos multiprofesionales en unidades básicas de salud, responsables del seguimiento de un número definido de familias, localizadas en un territorio delimitado. Se espera que los profesionales de salud puedan crear vínculos relativamente sólidos con las personas que viven en las áreas adscritas, lo que contribuiría a obtener mejores resultados en el control de enfermedades y en la producción de salud.

Con la inversión en atención primaria de la salud y la adopción de políticas públicas, como el Programa Más Médicos para Brasil, el Programa de Recalificación de Unidades Básicas de Salud y la creación de núcleos de apoyo a la salud familiar, entre otros, se ha buscado consolidar la Estrategia de Salud Familiar, principalmente, en lo que se refiere al aumento del acceso a los servicios de salud. 
Los equipos de salud familiar se componen de médicos, enfermeros, técnicos de enfermería, agentes comunitarios de salud, cirujano dentista y auxiliar de cirujano dentista y tienen bajo su responsabilidad territorios con una población entre tres y cuatro mil personas. El acceso de los habitantes al equipo de salud exige el registro y se da mediante consultas, participación en actividades grupales, visitas domiciliarias y procedimientos como vacunación y administración de medicamentos. Tomando en cuenta que, en muchas situaciones, el número de habitantes registrado para un equipo es mayor que lo recomendado, con el aumento de la pobreza y de las demandas de acciones en salud caracterizada por la alta complejidad social, gran parte de los equipos se encuentran sobrecargados y bajo estrés. Eso puede causar que se priorice la atención en la unidad de salud en detrimento de la asistencia en el territorio, de la misma manera, que hay una tendencia al cuidado de la enfermedad, aunque las demandas sociales sean evidentes.

\section{Ocupaciones urbanas}

Al contrario de las grandes favelas, ampliamente estudiadas, las ocupaciones urbanas recientes son fenómenos poco abordados desde la salud colectiva $^{(4)}$. La mayor parte de esa producción integra las áreas de arquitectura y urbanismo. La pandemia del nuevo coronavirus sacó a la luz esa discusión, sobre todo en relación con las áreas de más densidad poblacional y altamente vulnerables. Resulta evidente la necesidad de pensar de nuevo el trabajo en salud, especialmente, de la atención básica.

El Instituto Brasileño de Geografía y Estadística (IBGE) inscribe las ocupaciones urbanas en un gran grupo denominado "aglomeración subnormal" cuyas características son el aglutinamiento de viviendas fuera de los patrones urbanísticos, ubicados en áreas que son propiedad de otros (privado o público), y tienen servicios esenciales limitados. Están presentes en ese grupo otras formas de viviendas como favelas, palafitos, conventillos, etc. ${ }^{(5)}$.
Percibidas como ilegales y muy precarias, las ocupaciones urbanas recientes son áreas que antes fueron abandonadas y ahora transformados en lugares de vivienda por grupos de personas organizadas que buscan un mínimo de seguridad para sus familias, en la medida en que no poseen ingresos suficientes para pagar un alquiler y costear la alimentación, entre otras necesidades básicas. Ese escenario deriva, sobre todo, del aumento del desempleo y el valor de los alquileres ${ }^{(6)}$.

Rizzatti y Silva ${ }^{(4)}$ señalan que la acción de entrar al territorio se planea previamente y se da de manera rápida y colectiva. Debido a la necesidad de rapidez para construir las casas, los materiales utilizados son, generalmente, aquellos que facilitan ese proceso, como pedazos de madera y lonas. No se descartan las construcciones de ladrillo y se realizan con la intención de dificultar el reintegro de la posesión de la tierra.

Es interesante destacar que no todas las áreas ocupadas cuentan con el apoyo de movimientos institucionalizados en la lucha por las viviendas. Ciertas áreas están ocupadas por grupos de personas organizadas, pero sin vínculo con movimientos sociales. De ese modo, las formas organizativas en estos espacios son variadas.

Aunque el IBGE considere las viviendas localizadas en áreas pobres como aglomerados subnormales que no corresponden a las normas urbanísticas, existen diversas experiencias de viviendas que exceden sus formas físicas. En ese otro lado del habitar, la vida que transcurre por esos espacios no suele ser de conocimiento público, ni de los propios investigadores. Generalmente, los estudios se sustentan en presupuestos que ya no corresponden más a las realidades de esas localidades. En el caso de las favelas, aún son recurrentes tanto las imágenes retratadas en la década de 1940 cuyas formas físicas eran sobre todo barracas frágiles, como el cuestionamiento incisivo de la legitimidad de la posesión ${ }^{(7)}$.

Hablar sobre casas se constituye en una discusión compleja que va más allá de la simple concepción espacial y de las características generales de los domicilios. Como 
destaca Rolnik ${ }^{(8)}$, tener una casa está asociado a una serie de garantías de derechos, como acceso a los servicios públicos (colecta de basura, escuelas, salud, etc.), legitimidad del habitar, protección ante eventos climáticos, sumado a formas que no promuevan la discriminación de los que viven allí o impongan gastos que imposibiliten su permanencia.

Según Silva ${ }^{(9)}$, el entendimiento que tienen con relación a esas formas de habitar y de las personas que residen allí es lo que determina muchas veces el tipo de servicios ofrecidos. Al ser representadas a partir de imágenes que no están actualizadas y vistas por el sesgo de la subnormalidad, las acciones orientadas a esa parte de la población se realizan siempre desde el punto de vista de lo que debe ser ajustado.

De manera general, las medidas ofrecidas por el poder público son verticales y no acompañan las reales necesidades de los que viven allíl(10,11). Además de no considerar la subjetividad de las personas demandantes, algunas formas de mejoría no solo refuerzan estigmas, sino que contribuyen aún más a la cronicidad de la vulnerabilidad a la que están sujetos los individuos ${ }^{(11)}$.

El traslado de habitantes de áreas vistas como inadecuadas hacia otros lugares más aceptados son ejemplos de esta problemática. Santos ${ }^{(10)}$ destaca que las áreas legalizadas generalmente cuentan con servicios públicos y comercios que encarecen los espacios y dificultan la permanencia de los recién llegados. No es raro que esas familias regresen a las ocupaciones urbanas donde logran mantenerse. De ese modo, la oferta de vivienda o de servicios públicos no son suficientes para garantizar sus derechos ni abarcan la complejidad del problema.

En los permisos provisorios para registrar determinados terrenos, generalmente se les prohíbe a los habitantes construir o que realicen mejoras en esos espacios, dado que la posesión no está garantizada y el objetivo final es su remoción ${ }^{(12)}$. Se trata de prácticas similares a las implementadas en las formaciones de los conventillos del siglo pasado: las entidades gubernamentales se respaldaban en el discurso higienista que buscaba impedir la proliferación de enfermedades y de comportamientos considerados indolentes o vulgares y, en paralelo, afianzaba el proyecto de modernización de la porción central, que incluía la eliminación de las clases pobres ${ }^{(13)}$.

Al mantener a las personas en esa situación de insalubridad por tiempo indefinido pero prolongado, los órganos del Estado se vuelven responsables del riesgo al que están sujetos esos habitantes. Ciertos estudios demuestran que, en áreas como las ocupaciones urbanas, las personas lidian diariamente con temperaturas extremas, como mucho calor o mucho frío ${ }^{(14)}$, además de estar más expuestas a animales ponzoñosos o vectores de enfermedades, por situarse en áreas no tratadas y con acumulación de basura. Hay también riesgos de accidentes domésticos debido a la necesidad de reparaciones. La densificación, propia de esas formas de habitar, inviabiliza la privacidad y puede contribuir a desentendimientos entre vecinos ${ }^{(12,15)}$.

Esa inconstancia en la permanencia, así como la precariedad a la que están sujetos, afectan de distintas formas a esos habitantes. Según Kottow ${ }^{(16)}$, incluso entre las personas vulnerables, las desigualdades sociales coloca a ciertas personas en situaciones más vulnerables aún. En ese sentido, Helene ${ }^{(17)}$ señala que, en situaciones de viviendas precarias, los ancianos y las mujeres son los más afectados, en la medida en que pasan más tiempo en los domicilios y en sus inmediaciones, siendo más vulnerables a las insalubridades. Tal como se verá, las relaciones con la vivienda, así como las divisiones del trabajo, están marcadas por las desigualdades de género.

\section{Viviendas y mujeres}

La incertidumbre acerca de la vivienda afecta la salud de sus ocupantes, especialmente en el caso de las mujeres que poseen dependientes (hijos, padres mayores o enfermos) y que enfrentan diariamente las consecuencias de ser mujeres negras en una sociedad que es estructuralmente machista y racista, tornándolas aún más vulnerables. 
Frecuentemente, se asocia a las mujeres con el ámbito doméstico, en la medida en que su papel construido socialmente es el de cuidar la casa, la familia y a los otros, cuando trabajan afuera de sus casas. La esfera de lo privado y el cuidado a tiempo completo se perciben como naturalmente femeninos ${ }^{(17,18)}$.

Ese espacio restrictivo en cuanto al papel de la mujer se consolidó, sobre todo, con la representación de la familia nuclear y en la división sexual de trabajo. Debido a esa construcción social que atribuye a la mujer la función de cuidado, una postura comedida y poca participación en la vida pública, gran parte de sus acciones se desempeñan en el interior de sus hogares ${ }^{(18,19)}$. De ese modo, es esperable que la cuestión de la vivienda tenga una relevancia aún mayor en la vida de la mayoría de las mujeres, justamente, porque constituye parte de su identidad ${ }^{(17,20)}$.

En este sentido, residir en áreas consideradas ilegales es un factor de mayor preocupación para este grupo. Por un lado, existe el riesgo de desalojo, que provoca sufrimiento frente a la desprotección que impone la ausencia de un hogar, sobre todo ante la responsabilidad de garantizar la seguridad de sus dependientes ${ }^{(20)}$. Por otro lado, muchos hijos suelen ser desvinculados de sus madres debido a que las ocupaciones urbanas suelen ser concebidos como espacios peligrosos y precarios, y las capacidades de cuidado de las madres también suelen ser asociadas en esa dirección. Por lo tanto, la condición de la vivienda puede colocar a esas mujeres en situación de desventaja y sufrimiento, dado que constantemente deben dar pruebas de sus performances de cuidado.

De acuerdo con un estudio realizado en un ocupación urbana, las investigadoras Lyra y Kapp ${ }^{(21)}$ destacan que la preocupación de las habitantes es encontrar espacios que contemplen servicios destinados a sus dependientes, como escuelas y centros de salud, lo que va al encuentro de su papel de cuidadoras $^{(18)}$ y una concepción de la vivienda más allá de sus pilares estructurales ${ }^{(8)}$. Sin embargo, existen otras mujeres con otras demandas, además del papel predeterminado a partir de su sexo ${ }^{(21)}$. Paulista ${ }^{(20)}$ plantea que en situaciones de inseguridad habitacional, la casa, como ambiente de reclusión del sexo femenino, puede ser resignificada como espacio de lucha, donde esas mujeres se tornan líderes de la reivindicación del derecho a la vivienda.

Ante las demandas de los grupos organizados, los sectores públicos crean medidas para ese sector de la población. Sin embargo, tal como señalan Porto et al. ${ }^{(11)}$, esas decisiones son verticales, provisorias y amparadas en imágenes generalizantes.

En la intersección con la discusión de género, las medidas están tomadas sobre todo por hombres (que poseen un mayor número en cargos de gestión), amparados en normas urbanísticas que no aceptan la diversidad, en concepciones burguesas acerca de la composición familiar y de las necesidades de las mujeres $^{(17,21)}$.

Para la atención de la salud esa discusión es igualmente necesaria. Como señala Fernández ${ }^{(22)}$, los ambientes en los que viven y conviven las personas están más fuertemente relacionados con la producción-deterioro de la salud que sus hábitos o incluso sus predisposiciones genéticas.

Ciertos estudios relativos al sufrimiento psíquico y género indican que, para las mujeres, cumplir roles restrictivos y renunciar a sí mismas en vista del cuidado y los deseos del otro son factores que contribuyen al surgimiento de trastornos mentales comunes. En este sentido, también están presentes factores sociales como bajos ingresos, desempleos, ser negra, pocos años de escolaridad y escasos momentos de ocio ${ }^{(23)}$.

Las investigaciones centradas en la identificación de síntomas y su correlación con la pobreza y los hábitos considerados perjudiciales para la salud han reforzado los diagnósticos de trastornos psíquicos y la medicalización de la vida, sin tratar de las estructuras que los sustentan y las posibilidades de producción de salud en el escenario latinoamericano(24). Lo que producen esos estudios y muchos servicios de salud, según Zanello $^{(18)}$, es justamente, el silenciamiento de las desigualdades sociales. Aún son recurrentes las prácticas de responsabilizar a 
los sujetos por sus condiciones de salud, el no reconocimiento de su autonomía, además de la omisión de la magnitud que la determinación social tiene en el proceso salud-enfermedad-cuidado ${ }^{(25)}$.

En la compleja relación entre género, raza y vivienda, las formas más rígidas de cuidados terminan perdiendo de vista elementos muy importantes para el cuidado, incluso la potencia de los usuarios para responder sus propias inquietudes. En el caso de las mujeres, como ya están habituadas a ser juzgadas por el papel que desempeñan, evitan plantear sus necesidades o aspectos más íntimos en los servicios de salud, pudiendo ocultar, incluso, situaciones de violencia doméstica(18).

Las acciones de extensión universitaria desarrolladas en una ocupación urbana de la periferia de un municipio de gran tamaño, que se caracterizan por la interacción continua con los habitantes, indican que la forma organizativa de la atención primaria de la salud y el trabajo de equipos de salud familiar representan obstáculos para la comprensión adecuada de las diferentes facetas del sufrimiento de la parte más vulnerable de la sociedad. Se ha buscado aquí cotejar el discurso de mujeres que viven en la ocupación urbana y las formas de organización en la atención primaria de la salud.

\section{MÉTODO}

Se realizó una investigación cualitativa, exploratoria y transversal. Entre enero y febrero de 2020, se realizaron cuatro entrevistas en profundidad, semiestructuradas e individuales, que fueron grabadas y posteriormente transcritas integralmente. El proceso de elección de las entrevistadas incluyó la participación de una mujer, liderazgo con quien los investigadores mantenían un vínculo fuerte y a quien fue solicitado indicación de posibles participantes. Los criterios para la selección de esas mujeres eran: ser adultas, brasileñas, residentes durante al menos un año en la ocupación urbana referida. No se exigía que hubieran participado del programa de extensión.
Las participantes tenían entre 21 y 63 años y hacía al menos tres años que residían en el área ocupada. Todas eran madres, aunque apenas dos poseían hijos menores bajo sus cuidados. Para garantizar el resguardo de sus identidades se emplearon nombres ficticios. Las entrevistas se realizaron intencionalmente en las inmediaciones de la ocupación, en vista de la necesidad de un espacio de discreción y seguro para que ellas pudieran narrar vivencias que podrían generar incomodidad. Por el mismo motivo, se ha optado por encuentros individuales.

El inicio del diálogo se dio a partir de la construcción de sus genogramas, herramienta utilizada en atención primaria de la salud y en contextos terapéuticos diversos. Aunque parezca un árbol genealógico, debido a la representación gráfica familiar, el propósito del dibujo es pensar junto con las familias acciones en salud, intentando alcanzar aspectos además de las enfermedades, como las relaciones entre los componentes del grupo, fragilidades y potencialidades, aspectos culturales, entre otros. Valiéndose de una gama de símbolos estandarizados, señalan a las personas de la red y cómo se relacionan, a partir de líneas horizontales que unen una persona con otra, delineando la naturaleza de la unión. De ese modo, simultáneamente, mientras colocan las líneas en el dibujo, componen las narrativas ${ }^{(26,27)}$.

Como herramienta de investigación es igualmente potente, pues el sujeto elige dónde empezar, favoreciendo la comunicación entre el investigador y la persona participante. Al narrar su historia, la persona entrevistada recuerda elementos que le son significativos, eligiendo presentar $\mathrm{u}$ ocultar personas y acontecimientos ${ }^{(27)}$. Muchas veces, a través de la historia contada se puede alcanzar parte o totalmente los objetivos del estudio ${ }^{(26)}$.

Conforme contaban sus historias alrededor del núcleo familiar, las participantes se adentraban en otros terrenos y traían aspectos más íntimos. A través de la herramienta se puede explorar más a fondo la historia de vida de esas mujeres, sus caminos recorridos hasta alcanzar uno de los puntos de intersección: la ocupación urbana. Se ha intentado 
también identificar sus redes sociales significativas. Las narrativas no fueron totalmente libres, sino que se propuso una oferta temática vinculada a relaciones familiares $y$ sociales, vivir en una ocupación urbana, generadores de sufrimiento y estrés y acciones para enfrentarlos.

Desde el punto de vista metodológico, la utilización del genograma, más allá de afirmar de nuevo la completitud y potencia de la herramienta para acciones en salud, también posibilitó penetrar en la complejidad de esas vivencias, ofreciendo informaciones acerca del cuidado propuesto por la atención primaria de la salud.

Para el análisis de los datos se utilizó la técnica de análisis de contenido ${ }^{(28,29)}$, que ha considerado no solo el material de las entrevistas, sino la experiencia previa de los investigadores en el campo de estudio a partir del programa de extensión, además de las producciones científicas generadas por ese núcleo. El programa de extensión mencionado está destinado a una población muy pobre residente en una ocupación urbana reciente, y está bajo la responsabilidad de un equipo de salud familiar de una unidad cercana. En ese territorio viven aproximadamente 500 personas, con predominio de niños y adultos jóvenes. Los investigadores están insertos en este territorio hace más de tres años, realizando acciones de extensión universitaria lo que les permitió conocer el modo de vida de esa población.

Para la exploración y codificación del material se llevaron a cabo las siguientes etapas: lectura exhaustiva del conjunto de las narrativas, identificación de unidades de análisis y separación en temáticas con base en los objetivos del estudio, teorías que apoyan e impresiones de los autores. Esa selección temática tomó en cuenta las repeticiones de contenidos en las entrevistas, además de aquellos considerados de gran relevancia, aunque no estuvieran expresados en todas las conversaciones. Luego, se demarcaron las siguientes categorizaciones: provisoriedad, performances de género y trabajo en salud. Esa última fue una esfera transversal a todas las categorías y su discusión se hallará en intersección con las demás.
El proyecto fue aprobado por el Comité de Ética en Investigación (número del parecer 20093119.1.0000.5404). Los participantes aceptaron participar en el estudio, luego de que se les informara sobre la naturaleza de la investigación, sus objetivos y métodos, el beneficio esperado, los riesgos potenciales y las molestias que esta pudiera ocasionar. Los voluntarios recibieron una garantía de que sus identidades se mantendrán confidenciales y recibieron copia original del formulario de consentimiento firmado por el investigador.

\section{RESULTADOS Y DISCUSIÓN}

Cada trama presenta, de manera muy única, las vivencias de las mujeres en el contexto habitacional. No obstante, únicas y singulares, las narrativas contaron también con experiencias que parecen ser compartidas por otras mujeres que viven allí. Los eventos causantes de sufrimientos están fuertemente cimentados en las desigualdades sociales. Las entrevistadas relataron trayectorias marcadas por la violencia intrafamiliar extrema, pobreza, redes significativas diminutas y salida de la casa de los padres a temprana edad. Esa partida antes de cumplir los 15 años, estaba relacionada a la unión con compañeros de mayor edad y búsqueda por una vida mejor.

Un estudio similar reveló que las mujeres ya se encontraban en posiciones muy vulnerables antes del traslado a la ocupación urbana $^{(30)}$. Tales resultados apuntan a una posible vulnerabilidad crónica que acompaña a esta parte de la población ante la imposibilidad de poseer una vivienda. A continuación, se presentan y discuten las categorías de análisis de este estudio.

\section{Provisoriedad y salud}

Para muchas mujeres tener una casa, aunque sea precaria, puede significar una conquista, protección y salida de condiciones aún peores $^{(30)}$. Si bien, por un lado, esas viviendas posibilitan nuevas maneras organizativas y 
redes de cuidado ${ }^{(17,31)}$, por otro, pueden perpetuar la exclusión social y las condiciones de vida muy precarias, principalmente, en el caso de personas viviendo en ocupaciones urbanas recientes.

La provisoriedad fue señalada como uno de los principales temores y generadores de sufrimientos para las personas que viven en esos espacios. El sufrimiento surge del miedo a los desalojos (muy violentos), a la precariedad de la vida diaria, tal como se puede observar en los siguientes relatos:

Noelle: Hay luz, ¿sabes? Pero se vive cortando, y se descongela la heladera, se quema un aparato aquí, otro allá.

Adriana: Agarraba mucho balde [para traer agua], ya llevé muchos baldes, pero enseguida compramos una manguera larga. Ahí abrís la manguera y ya llenás las cosas, y después yo lo cierro, pero hay mucha gente que agarra con el balde.

Esos fragmentos explicitan que, aunque la presencia del Estado garantice algunos servicios básicos, son transitorios, insuficientes y no eliminan los riesgos a la salud. Según Rolnik ${ }^{(32)}$ el acto de ceder terrenos y servicios no es nuevo o exclusivo de Brasil, y tiene como fondo el mantenimiento de una relación de dependencia por parte de los vulnerables.

Las acciones del Estado no garantizan derechos, sino que mantiene la estructura de exclusión social, contribuyen al sentimiento de no pertenecer y pueden disgregar la lucha por un objetivo común ${ }^{(11)}$. También, para las participantes de este estudio, vivir en esas condiciones tiene implicaciones en sus subjetividades. En este caso, se observaron experiencias de disminución de la inversión en el propio ambiente:

María Flor: Mirá, tu cama se rompe, no podés comprar una cama nueva para poner ahí, ¿si se prende fuego? [risas] [...] Me quedo parada en el tiempo.

Noelle: Sí, yo siento, porque es madera [la vivienda], el piso es de tierra. Entonces, no tengo cómo arreglarlo como yo quiero: colocar piso, las cosas así. [Entrevistadora: ¿no puedes?]. No, como poder, puedo, pero viviendo ahí no me da ganas de poner el piso, arreglar todo bonito.

Esa condición de lo provisorio impide que las participantes ejecuten mejoras en sus viviendas y en los otros segmentos del territorio. La no propiedad, incluso con el permiso de ocupación por tiempo indeterminado, hace que esas personas se vean en un campo de inestabilidad prolongada que dificulta la potencia de acción y la creación de proyectos futuros ${ }^{(21,32)}$.

La provisoriedad también contribuye a que muchas de las cuestiones, como las presentadas en ese estudio, no lleguen a los equipos de salud. Al estar hace poco tiempo en el territorio, su vínculo es más frágil con los profesionales de referencia, algo fundamental para el ejercicio de la clínica ampliada y compartida o centrada en la persona ${ }^{(33,34)}$.

En general, el seguimiento de esta población es menor por la dificultad de acceso a la unidad de salud. Un estudio realizado en el mismo territorio ${ }^{(35)}$ reveló dificultad, resistencia y morosidad del proceso de incorporación de esa área de ocupación urbana al equipo de salud familiar a causa de la sobrecarga de trabajo, que ocasionó una restricción en el acceso a las acciones de salud. Otra investigación similar ${ }^{(31)}$, realizada en un ocupación urbana en el estado de Minas Gerais, también muestra la dificultad en garantizar el acceso a la salud para aquellos que viven en áreas recién ocupadas. En ambos estudios, uno de los impedimentos para dar acogida a esas familias fue justamente la no posesión de una vivienda, lo que inviabilizó el cuidado en salud de los residentes de ocupaciones urbanas recientes y consideradas ilegales. La discusión acerca de la incorporación de grandes áreas a los equipos de salud es compleja e involucra a diferentes niveles de la gestión. Sin embargo, la demora de esos procesos dejan sin cobertura a las personas más vulnerables ${ }^{(35)}$.

Otra de las razones por las que estas cuestiones relacionadas con el sufrimiento 
no llegan a los equipos, se relaciona con los desplazamientos en busca de cuidado que realizan los habitantes de ocupaciones urbanas recientes, por el vínculo que mantienen con la unidad de atención de salud a la que asistían antes. Conservar esa relación, implica un sentimiento de pertenencia con el antiguo territorio, un vínculo generacional con determinado profesional y el recelo de estar en una vivienda provisoria y no tener un hogar fijo de cuidado.

Los entrecruzamientos entre las redes de asistencia no son infrecuentes y fueron observados por otros investigadores. Según Cecílio et al. ${ }^{(36)}$, ante las dificultades de acceso, los usuarios de los servicios de salud buscan otros caminos en la red de asistencia en paralelo a los procesos institucionalizados, que apuntan a resolver sus demandas y trazan nuevos diseños en las redes de cuidado. Esos desplazamientos, también denominados como "actuar lego", muchas veces no son reconocidos por los profesionales como saber inventivo de los usuarios, con potencial de crear posibles soluciones a desafíos ya enfrentados por la gestión. La propuesta de los autores es que el sistema acoja esos nuevos "mapas de cuidado" como una opción para la producción de salud que involucre a todos los actores. Para aquellos que sobreviven en un territorio provisorio, como los habitantes de ocupaciones urbanas recientes, el reconocimiento de esas prácticas y de la necesidad de cambios paradigmáticos es indispensable y urgente.

Como señalan Cecílio et al. ${ }^{(36)}$, para algunos sujetos existen innumerables elementos que influyen $y$, a veces, dificultan, trazar caminos de cuidado y encontrar medios para enfrentar los desafíos vividos diariamente. Esas cuestiones deben ser reconocidas por usuarios y profesionales y trabajadas en conjunto.

\section{Performances de género y sufrimiento: consideraciones necesarias}

Las performances de género, que delimitan scripts de acuerdo con el sexo de las personas, pueden promover posturas diferentes con relación al cuidado en salud y el desencuentro entre profesionales y usuarias. Para los hombres, por ejemplo, no es común asistir a centros de salud o buscar prácticas de autocuidado ${ }^{(18)}$. Para las mujeres de este estudio, cumplir roles de género representó desafíos para las prácticas de cuidado.

Como ya se ha mencionado, la identidad de las mujeres es construida a partir de sus roles de cuidado de la familia y del hogar, incluso después de los cambios significativos a partir de las luchas feministas ${ }^{(18)}$. Las entrevistadas señalaron la diferencia entre la relación de los hombres que habitan en la ocupación con el espacio público y su propio vínculo. De acuerdo al relato de las mujeres, ellas pasan parte de sus días dentro de sus casas, con poco contacto con el resto de los vecinos. Esa experiencia de aislamiento fue señalada como promotora del sufrimiento.

El aislamiento cotidiano apunta a evitar desacuerdos con vecinos o compañeros, miedo que no está presente en ellos, pues "para la mujer es diferente". Una de las medidas de silenciamiento es justamente desalentar la vida pública bajo la justificación de que son las mujeres las responsables de generar desavenencias causantes de conflictos $^{(19)}$.

Además del aislamiento, otras formas de silencio y, potencialmente generadoras de sufrimiento, fueron observadas durante las entrevistas. El relato sobre sus mecanismos para enfrentar el estrés diario y las formas de sufrimiento incluyó frases como las siguientes:

Fernanda: Guardo [...]. Hay cosas que guardamos para nosotros mismos. Ahí se queda, y después pasa.

Adriana: Me quedo callada, ¿sabés? Me quedo en silencio. Guardo todo dentro de mí, es así, me quedo más callada, cierro la cara [risas] [...] Guardo la rabia dentro de mí, entonces pasa.

Maria Flor: Soy muy sentimental, guardo muchas cosas aquí dentro.

Noelle: No hago nada. A veces me quedo en silencio para no hablar demás, para 
no lastimarlo [compañero]. Las cosas que digo son muy pesadas, entonces me callo.

El silencio, en ese sentido, aparece como algo natural y la medida para lidiar con el sufrimiento, evitando compartir con otros, desde la perspectiva que le cabe a ella, a la mujer, el papel de cuidar. En esa visión performática de género, el rol de una buena mujer es evitar traerle problemas a los compañeros bajo el riesgo de ser calificadas como problemáticas, sustituidas por otras mujeres o, incluso, agredidas $^{(18)}$.

Cuando se habitúan al aislamiento y al silencio, esas mujeres no buscan los servicios de salud u otras redes de apoyo y, cuando ocurre, hay una retracción por el miedo de ser juzgadas por desempeñar mal su rol ${ }^{(18)}$. En otros casos, como en situaciones de violencia doméstica, el silencio también viene acompañado del miedo por el agresor y la desconfianza en cuanto al sistema de protección ofrecido ${ }^{(37,38)}$.

La neutralización del silencio (desde muy temprana edad), hace que otros comportamientos desviantes de la performance femenina requerida no sean percibidos ni por ellas, ni por profesionales de la salud como mecanismos para enfrentar sus cotidianos y puedan ser entendidos como problemas psíquicos, por ejemplo, las conductas consideradas agresivas o lloro constante ${ }^{(18)}$.

A pesar de los avances en la atención integral a la salud de la mujer, la relación entre trabajadores de la atención primaria de la salud y las mujeres se establece más frecuentemente a partir del cuerpo femenino vinculado a la sexualidad, la reproducción, el climaterio y las neoplasias. El sufrimiento constatado en las mujeres en situación de gran vulnerabilidad demora más en ser detectado $y$, en general, es cuando ya no puede ser silenciado. En el caso de violencia doméstica, Silva et al. ${ }^{(39)}$ destacan cómo las situaciones de violencia psicológica se quedan ocultas en detrimento de las físicas, pues son formas de sufrimientos menos aparentes.

Sin embargo, si bien hay una revalorización de la práctica en salud orientada al sujeto, como la propuesta de la clínica ampliada y compartida y el método clínico centrado en la persona, sigue siendo hegemónico el método tradicional basado en la anamnesis y el raciocinio patofisiológico que tiene por objeto la enfermedad. Eso dificulta mirar otras necesidades. La presencia de cuestiones relacionadas con el sufrimiento de género y la desigualdad social en los encuentros clínicos, depende de una postura intencional y activa de los profesionales de salud, en el sentido de ampliar el alcance de su prácticas. Según las mujeres entrevistadas:

María Flor: Nunca nadie habla de esos temas con nosotros y siempre queda bajo siete llaves, guardado allá.
Adriana: Voy al centro de salud, pero no es solo para esas cosas, tampoco así. Es muy difícil conseguir una consulta para nosotros y cuando la conseguimos, la atención es un poco incierta.

Si bien existe una vasta producción académica en el campo del trabajo en salud en atención primaria y Estrategia de Salud Familiar, con responsabilidad sanitaria por el territorio y la poblaciones definidos, lo que se ha observado durante las acciones de extensión universitaria longitudinales y continuas y a partir de los relatos de las mujeres entrevistadas fue la existencia de un sufrimiento "invisible", casi imperceptible para los trabajadores de la salud, sobrecargados y rehenes de procesos de trabajo que los consume dentro de las unidades de salud y de la biomedicina.

Los núcleos de apoyo a la salud familiar de la región brindan soporte a siete equipos de salud familiar distribuidos en dos unidades básicas de salud. Los profesionales participan de las reuniones de los equipos y contribuyen en la discusión de casos, la elaboración de proyectos terapéuticos singulares, realizan consultas individuales y compartidas, visitas domiciliarias y grupos educativos y terapéuticos. El núcleo de apoyo a la salud familiar de la región fue capaz de ampliar el objeto de la clínica en atención primaria; sin embargo, si el núcleo está distante de los territorios 
vulnerables, su trabajo pasa a estar muy "pautado" por los equipos de salud familiar que dejan de relacionar a quienes residen en ocupaciones urbanas con estos profesionales que tendrían más posibilidad de ampliar la clínica de los propios profesionales de la salud familiar.

El ejercicio de comprensión de las formas de sufrimiento y resistencia de las mujeres que viven en una ocupación urbana reciente, conjuntamente al trabajo realizado en el territorio a través del programa de extensión universitaria aportaron relatos y observaciones interesantes que suscitaron reflexiones acerca de la organización de la atención primaria de la salud, especialmente en la relación del trabajo en salud con grupos muy vulnerables. Las entrevistas realizadas revelaron condiciones de sufrimientos que no suelen aparecer en el marco de la atención realizada por los profesionales de los equipos, habituados a consultas en unidades básicas de salud o visitas domiciliares puntuales.

Además del desafío planteado por la aparición repentina de nuevas familias debido a nuevas ocupaciones territoriales -algo que, según las tendencias, se tornará cada vez más recurrente ${ }^{(4,5,40)}$ - hay también formas organizativas que recluyen a los profesionales de atención primaria, y obstruyen sus canales de recepción y reducen su potencialidad de actuar más allá de tecnicismos ${ }^{(41)}$.

Las ocupaciones urbanas recientes, al demandar configuraciones que no se condicen con las normativas, pueden posibilitar espacios de ampliación de la autonomía de sus habitantes, además de estar más propensos a la creatividad. Aunque en un número restringido, hay estudios que abordan experiencias potentes al interior de esos espacios ${ }^{(17,20,31)}$. Esas experiencias produjeron nuevas maneras de habitar, relacionarse y cuidar, y se sustentan, sobre todo, en la acogida, la lucha común, la distribución de responsabilidades y el cuidado individual y colectivo, que según Czeresnia ${ }^{(42)}$, son fuertes componentes para el fortalecimiento de la salud. A pesar de no ser el objetivo de este estudio, es importante destacar que en la ocupación urbana también se observaron tales prácticas, siendo ejemplos de ello el uso creativo de las redes de cuidado y la propia lucha por la vivienda. Esos individuos y colectivos poseen saberes sobre el cuidado y su propio territorio que pueden tener mucho para ofrecer a los equipos de salud, especialmente, en la relación con la corresponsabilización del proceso salud-enfermedad-cuidado.

Adentrarse más en el territorio y promover otros posibles desplazamientos podría ser un modo de tejer nuevas redes en conjunto con los usuarios, especialmente, porque ellos tienen el privilegio de ser la autoridad en esos espacios. Esto no impide intentar que el trabajo sea más relacional, la imprevisibilidad propia de la vida en curso dificulta las acciones puramente tecnicistas y exige disposición para reinventar procesos de cuidado $^{(41)}$.

\section{CONCLUSIÓN}

Los temas planteados en las entrevistadas imponen algunas cuestiones sobre cómo está organizado el proceso de trabajo en atención primaria. Trascurridos más de 25 años del inicio de la implantación de la Estrategia de Salud Familiar en el país conviene reflexionar sobre su real alcance en el sentido de responder a las demandas de la sociedad, principalmente, en un contexto de aumento reciente de la pobreza, desempleo e iniquidad, y riesgo de disminución de la participación del Estado, en particular, después del crecimiento político de la derecha y la pérdida de espacio de la izquierda en el país.

Los hallazgos de este estudio señalan la necesidad de fortalecer las acciones en el territorio y, por lo tanto, fuera de los límites de las unidades básicas de salud, del trabajo multiprofesional e interdisciplinar y de acciones intersectoriales.

Proponemos colocar en la agenda la revisión y perfeccionamiento de la Estrategia de Salud Familiar, principalmente en lo que se refiere a la formación de profesionales, al modo cómo se interrelacionan y sus atribuciones. 


\section{AGRADECIMIENTOS}

A la Coordenação de Aperfeiçoamento de Pessoal de Nível Superior (CAPES), proceso No. 88882.434619/2019-01, por el apoyo ofrecido en forma de beca de maestría, otorgada desde septiembre de 2019 a junio de 2021.

\section{REFERENCIAS BIBLIOGRÁFICAS}

1. Jornal da USP. Situação dramática do desemprego na pandemia está oculta nos indicadores oficiais [Internet]. 2020 [citado 2 jul 2020]. Disponible en: https://tinyurl. com/f9c4dtfc.

2. Oxfam Brasil. Recompensem o trabalho, não a riqueza [Internet]. 2018 [citado 22 nov 2020]. Disponible en: https://tinyurl.com/ymdaxhub.

3. Travassos C, Oliveira EXG de, Viacava F. Desigualdades geográficas e sociais no acesso aos serviços de saúde no Brasil: 1998 e 2003. Ciência \& Saúde Coletiva. 2006;11(4):975-986. doi: 10.1590/S141381232006000400019 .

4. Rizzatti H, Silva AMB. Cidades e contra-racionalidades: Ocupações urbanas em Campinas/SP (Do Parque Oziel ao Jardim Campo Belo). Raega - O Espaço Geográfico em Análise. 2017;40:211-230.

5. Instituto Brasileiro de Geografia e Estatística. Censo demográfico 2010: Aglomerados subnormais - informações territoriais [Internet]. 2013 [citado 10 jun 2020]. Disponible en: https://tinyurl.com/3ahsp3ub.

6. Boulos G. Por que ocupamos? Uma introdução à luta dos sem-terra. São Paulo: Scortecci; 2012.

7. Silva MLP. Favela: É geral? É particular? É urbano? En: Silva JS, Barbosa JL, Biteti MO, Fernandes FL, eds. O que é a favela, afinal? [Internet]. Rio de Janeiro: Observatório de Favelas do Rio de Janerio; 2009. p. 30-35 [citado 10 jun 2020]. Disponible en: https://tinyurl.com/2nm2xa8u.

8. Rolnik R. Moradia é mais que um objeto físico de quatro paredes. e-metropolis. 2011;(5):37-42.

9. Silva JS. O que é favela, afinal? En: Silva JS, Barbosa JL, Biteti MO, Fernandes FL, eds. O que é favela, afinal? [Internet]. Rio de Janeiro: Observatório de Favelas do Rio de Janerio; 2009. p. 21-23 [citado 10 jun 2020]. Disponible en: https://tinyurl.com/2nm2xa8u.

10. Santos M. Ocupação periférica e reprodução do centro. En: Metrópole corporativa fragmentada: o caso de São Paulo. São Paulo: Nobel/Secretária de Estado da Cultura; 1990. p. 37-74.

11. Porto MFS, Cunha MB, Pivetta F, Zancan L, Freitas JD. Saúde e ambiente na favela: reflexões para uma promoção emancipatória da saúde. Serviço Social \& Sociedade. 2015;(123):523-543. doi: 10.1590/01016628.035 .

12. Castelaneli IKM, Vilela MFG, Bedrikow R, Santos DS, Figueira MCS. Na ausência de endereço, onde mora a saúde? Determinantes sociais e populações de ocu- pações. Saúde em Debate. 2019;43(spec 8):11-24. doi: 10.1590/0101-6628.035.

13. Vaz LF. Dos corticos as favelas e aos edificios de apartamentos: a modernizacao da moradia no Rio de Janeiro. Análise Social. 1994;29(127):581-597.

14. Silva EN, Ribeiro H. Alterações da temperatura em ambientes externos de favela e desconforto térmico Temperature modifications in shantytown environments and thermal discomfort. Revista de Saúde Publica. 2006;40(4):663-670. doi: 10.1590/S003489102006000500016.

15. Pasternak S. Habitação e saúde. Estudos Avançados. 2016;30(86):51-66

16. Kottow M. Bioética de proteção: considerações sobre o contexto latino-americano. En: Schramm FR, Rego S, Braz M, Palácios M, eds. Bioética: riscos e proteção. Rio de Janeiro: UFRJ/Fiocruz; 2005. p. 29-44.

17. Helene D. Gênero e direito à cidade a partir da luta dos movimentos de moradia. Cadernos Metrópole. 2019;21(46):951-974. doi: 10.1590/2236-9996.20194612.

18. Zanello V. Saúde mental, gênero e dispositivos: cultura e processos de subjetivação. Curitiba: Appris; 2018.

19. Perrot M. As mulheres ou os silêncios da história. Bauru: EDUSC; 2005

20. Paulista A. As mulheres nos movimentos sociais de moradia: a cidade sob uma perspectiva de gênero. Humanidades em Diálogo. 2013;5:93-108. doi: 10.11606/ issn.1982-7547.hd.2013.106242.

21. Lyra L, Kapp S. Mulheres em outra moradias. Revistas Periferias [Internet]. 2019 [citado 10 jun 2020]. Disponible en: https://tinyurl.com/2wmr6ter.

22. Fernández RC. Tú código postal es más importante para tu salud que tu código genético (1). Atención Primaria. 2013;45(3):127-128. doi: 10.1016/j. aprim.2013.02.001

23. Zanello V, Fiuza G, Costa HS. Saúde mental e gênero: facetas gendradas do sofrimento psíquico. Fractal: Revista de Psicología. 2015;27(3):238-246. doi: 10.1590/1984-0292/1483.

24. Gama CAP, Campos RTO, Ferrer AL. Saúde mental e vulnerabilidade social: a direção do tratamento. Revista de Latinoamericana de Psicopatología Fundamental. 2014;17(1):69-84. doi: 10.1590/S141547142014000100006 .

25. Barata RB. Como e por que as desigualdades sociais fazem mal à saúde. Rio de Janeiro: Editora Fiocruz; 2009.

26. Cardim MG, Moreira MCN. Adolescentes como sujeitos de pesquisa: a utilização do genograma como apoio para a história de vida. Interface - Comunicação, Saúde, Educação. 2013;17(44):133-143. doi: 10.1590/ S1414-32832013000100011. 
27. Krüger LL, Werlang BSG. O genograma como recurso no espaço conversacional terapêutico. Avaliação Psicológica. 2008;7(3):415-426.

28. Bardin L. Análise do conteúdo. Edições 70. São Paulo; 2011.

29. Campos CJG. Método de análise de conteúdo: ferramenta para a análise de dados qualitativos no campo da saúde. Revista Brasileira deEnfermagem. 2004;57(5):611614. doi: 10.1590/S0034-71672004000500019.

30. Brandão TO, Germando IMP. Experiência, memória e sofrimento em narrativas autobiográficas de mulheres. Psicologia \& Sociedade. 2009;21(1):5-15. doi: 10.1590/ S0102-71822009000100002.

31. Almeida SP, Torres LM, Simim DA, Paula PP, Souza NM. Percepção dos moradores de uma ocupação urbana sobre o 'empoderamento' em saúde. Saúde em Debate. 2020;44(125):335-348. doi: 10.1590/01031104202012504

32. Rolnik R. Os sem lugar ou a crise global de insegurança da posse. En: Rolnik R, ed. Guerra de lugares: a colonização da terra e da moradia na era das finanças. São Paulo: Boitempo; 2015.

33. Campos GWS. Saúde Paidéia. São Paulo: Hucitec; 2003.

34. Lopes JMC, Dias LC. Consulta e abordagem centrada na pessoa. En: Gusso G, Lopes JMC, Dias LC, eds. Tratado de medicina de família e comunidade: princípios, formação e prática. Porto Alegre: Artmed; 2012.

35. Sestari CZ, Vilela MF de G, Bedrikow R. Incorporação de Aglomerado Subnormal à uma Equipe de Saúde da
Família. Revista dos Trabalhos de Iniciação Científica da UNICAMP. 2019;(27). doi: 10.20396/revpibic.

36. Cecilio LCO, Carapinheiro G, Andreazza R, Souza ALM, Andrade MGG, Santiago SM, et al. O agir leigo e o cuidado em saúde: a produção de mapas de cuidado. Cadernos de Saúde Pública. 2014;30(7):1502-1514. doi: 10.1590/0102-311X00055913.

37. Dutra ML, Prates PL, Nakamura E, Villela WV. A configuração da rede social de mulheres em situação de violência doméstica. Ciência \& Saúde Coletiva. 2013;18(5):1293-1304. doi: 10.1590/S141381232013000500014

38. Moré CLOO, Krenkel S. Violência no contexto familiar. Florianópolis: UFSC; 2014.

39. Silva LL, Coelho EBS, Caponi SNC. Violência silenciosa: violência psicológica como condição da violência física doméstica. Interface - Comunicação, Saúde, Educação. 2007;11(21):93-103. doi: 10.1590/S141432832007000100009 .

40. Pasternak S, D'Ottaviano C. Favelas no Brasil e em São Paulo: avanços nas análises a partir da Leitura Territorial do Censo de 2010*. Cadernos Metrópole. 2016;18(35):75100. doi: 10.1590/2236-9996.2016-3504.

41. Franco TB, Merhy EE. Cartografias do Trabalho e Cuidado em Saúde. Tempus Actas de Saúde Coletiva. 2012;6(2):151-163. doi: 10.18569/tempus.v6i2.1120.

42. Czeresnia D. O conceito de saúde e a diferença entre prevenção e promoção. En: Czeresnia D, ed. Promoção da saúde: conceitos, reflexões, tendências. Rio de Janeiro: Editora FIOCRUZ; 2003. p. 39-54.

FORMA DE CITAR

Mota AM, Bedrikow R. Sufrimiento de mujeres en una ocupación urbana de São Paulo, Brasil: un desafío para el trabajo en atención primaria de la salud. Salud Colectiva. 2021;17:e3358. doi: 10.18294/sc.2021.3358.

Recibido: 18 dic 2020 | Versión final: 10 mar 2021 | Aprobado: 22 mar 2021 | Publicado en línea: 3 jun 2021

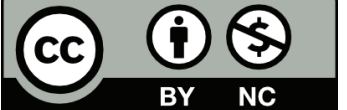

Esta obra está bajo una licencia de Creative Commons Reconocimiento-NoComercial 4.0 Internacional. Reconocimiento - Permite copiar, distribuir y comunicar públicamente la obra. A cambio, se debe reconocer y citar al autor original. No Comercial - Esta obra no puede ser utilizada con finalidades comerciales, a menos que se obtenga el permiso. 